\title{
TRANSCENDENTAL NUMBERS WITH BADLY DISTRIBUTED POWERS ${ }^{1}$
}

\author{
DAVID W. BOYD
}

In this paper we shall construct an uncountable set of numbers which exhibit to some extent the unusual properties of the classes of algebraic integers studied by Pisot, Vijayaraghavan and Salem (see $[1],[3],[5],[6])$. We denote by $T$ the set of real algebraic integers $\theta>1$ such that all conjugates of $\theta$ lie within or on the unit circle. As usual $\|x\|$ will denote the distance from the real number $x$ to the nearest in teger. If $\theta \in T$, and $\epsilon>0$ is arbitrary then there are numbers $\lambda$ in the field $Q(\theta)$ such that $\left\|\lambda \theta^{n}\right\| \leqq \epsilon$ for $n=0,1,2, \ldots$ (see [6]; combine Theorem 2, p. 3 with the argument on p. 33). On the other hand, a result due to Pisot [3] states that if $\theta>1, \lambda \geqq 1$ are real and such that

$$
\left\|\lambda \theta^{n}\right\| \leqq(2 e \theta(\theta+1)(1+\log \lambda))^{-1} \quad n=0,1,2, \cdots
$$

then $\theta \in T, \operatorname{deg} \theta \leqq[\log \lambda]+1$, and $\lambda \in Q(\theta)$.

It is reasonable to ask how much this result can be improved by increasing the right member of (1), and still obtaining the conclusion that $\theta$ is algebraic. Our construction will show that there are arbitrary large transcendental $\theta>3$, and $\lambda$ arbitrarily close to 2 such that

$$
\left\|\lambda \theta^{n}\right\| \leqq(\theta-1)^{-1}(\theta-3)^{-1} \quad \text { for } n=0,1,2, \cdots .
$$

Thus, for example $\left\|\lambda \theta^{n}\right\| \leqq 10(2 e \theta(\theta+1)(1+\log \lambda))^{-1}$ for all $n \geqq 0$, need not imply that $\theta$ is algebraic, (see the remarks following Theorem1).

Pairs $(\lambda, \theta)$ satisfying (2) are quite rare, for Weyl [7] shows that, for fixed $\theta$, the sequence $\left\{\lambda \theta^{n}\right\}$ is uniformly distributed modulo 1 for almost all real $\lambda$, and Koksma [2] shows that, for fixed $\lambda$, the sequence $\left\{\lambda \theta^{n}\right\}$ is uniformly distributed modulo 1 for almost all real $\theta>1$. However, if $\lambda$ and $\theta$ satisfy (2), $\left\{\lambda \theta^{n}\right\}$ is not even dense modulo 1 .

Our main result is the following:

Theorem 1. Let $\alpha, \beta$ be real numbers with $3<\alpha<\beta$, and let $a_{0}$ be an integer satisfying $a_{0}>(\alpha+1)(\alpha-1)^{-1}(\beta-\alpha)^{-1}$. Then there is an uncountable set $S \subset[\alpha, \beta]$, such that for each $\theta \in S$, there is a real number $\lambda=\lambda(\theta)>0$ for which

$$
\left\|\lambda \theta^{n}\right\| \leqq(\alpha-1)^{-1}(\theta-1)^{-1} \quad \text { for } n=0,1, \cdots .
$$

The integer $a_{0}$ is the nearest integer to $\lambda(\theta)$ for all $\theta \in S$.

Received by the editors March 19, 1969.

1 This work was supported in part by NSF grant GP 6111. 
Proof. We construct each element in the set $S$ as the limit of a sequence $\left\{a_{n+1} / a_{n}\right\}$ where each $a_{n}$ is a positive integer. To insure that $S$ is uncountable we obtain a one-one correspondence between $S$ and the set $J$ of functions mapping $Z^{+} \rightarrow\{0,1\}$, (here $Z^{+}$ $=\{1,2, \cdots\}$ ).

To begin with, we note that $a_{0}>(\alpha+1)(\alpha-1)^{-1}(\beta-\alpha)^{-1}$ implies that

$$
\left(a_{0} \beta-(\alpha-1)^{-1}\right)-\left(a_{0} \alpha+(\alpha-1)^{-1}\right)>1 .
$$

Thus there is an integer $a_{1}$ with

$$
a_{0} \alpha+(\alpha-1)^{-1}<a_{1}<a_{0} \beta-(\alpha-1)^{-1} .
$$

Let $a_{1}$ be any such integer (say the smallest). Now, if $f \in J$, we define a sequence $\left\{a_{n}(f)\right\}$ of integers by

$$
\begin{gathered}
\quad a_{0}(f)=a_{0}, \quad a_{1}(f)=a_{1}, \\
a_{n+1}(f)=\left[a_{n}^{2}(f) / a_{n-1}(f)\right]+f(n), \quad n=1,2, \cdots,
\end{gathered}
$$

where as usual the square brackets denote the integer part.

Now define $\rho_{n}(f)=a_{n}(f) / a_{n-1}(f)$. For the moment we shall write $a_{n}=a_{n}(f), \rho_{n}=\rho_{n}(f)$. Note that (5) implies

$$
\left|\rho_{n+1}-\rho_{n}\right| \leqq a_{n}^{-1}, \quad n=1,2, \cdots
$$

Thus, we have

$$
\left|\rho_{n+1}-\rho_{1}\right| \leqq \sum_{k=1}^{n} a_{k}^{-1}=\sum_{k=1}^{n}\left(\rho_{k} \rho_{k-1} \cdots \rho_{1}\right)^{-1} a_{0}^{-1} .
$$

We wish to show by induction that $\alpha<\rho_{n}<\beta$ for all $n$. First observe that (4) implies $\alpha<\rho_{1}=a_{1} / a_{0}<\beta$. Assume that $\alpha<\rho_{n}<\beta$ for $k$ $=2,3, \cdots, n$. Then, from (7) we have

$$
\left|\rho_{n+1}-\rho_{1}\right|<a_{0}^{-1} \sum_{k=1}^{n} \alpha^{-k}<a_{0}^{-1}(\alpha-1)^{-1}
$$

and hence

$$
\rho_{1}-a_{0}^{-1}(\alpha-1)^{-1}<\rho_{n+1}<\rho_{1}+a_{0}^{-1}(\alpha-1)^{-1} .
$$

However, by (4), $\rho_{1}-a_{0}^{-1}(\alpha-1)^{-1}=\left(a_{1}-(\alpha-1)^{-1}\right), a_{0}^{-1}>\alpha$, and similarly the right member of (8) is less than $\beta$, so $\alpha<\rho_{n+1}<\beta$ completing the induction.

Again using (6), we can show $\left\{\rho_{n}\right\}$ is a Cauchy sequence, for if $n<m$, we have 


$$
\begin{aligned}
\left|\rho_{m}-\rho_{n}\right| & \leqq \sum_{k=n}^{m-1}\left|\rho_{k+1}-\rho_{k}\right| \\
& \leqq \sum_{k=n}^{m-1} a_{k}^{-1}<\sum_{k=n}^{m-1} \alpha^{-k+n-1} a_{n-1}^{-1}<a_{n-1}^{-1}(\alpha-1)^{-1}
\end{aligned}
$$

where we have used $a_{k}=\rho_{k} \rho_{k-1} \cdots \rho_{n} a_{n-1}>\alpha^{k-n+1} a_{n-1}$. Since $\alpha>3$, clearly $a_{n}>\alpha^{n} a_{0} \rightarrow \infty$ so (9) shows that $\left\{\rho_{n}\right\}$ is a Cauchy sequence hence converges to $\theta=\theta(f)$, say, with $\alpha \leqq \theta \leqq \beta$.

We next show that if $f, g \in J, f \neq g$, then $\theta(f) \neq \theta(g)$. For, if $f \neq g$, let $f(k)=g(k)$ for $k=1,2, \cdots, n-2$ and $f(n-1)>g(n-1)$ so $f(n-1)$ $=1, g(n-1)=0$. Then $a_{k}(f)=a_{k}(g)$ for $k=1,2, \cdots, n-1$, and $a_{n}(f)=a_{n}(g)+1$. Thus

$$
\rho_{n}(f)-\rho_{n}(g)=\left(a_{n}(f)-a_{n}(g)\right) / a_{n-1}(f)=a_{n-1}^{-1}(f) .
$$

But, for $k \geqq n$,

$$
\begin{aligned}
& \left|\rho_{k+1}(f)-\rho_{k+1}(g)\right| \\
& \quad \geqq\left|\rho_{k}(f)-\rho_{k}(g)\right|-\left|\rho_{k+1}(f)-\rho_{k}(f)\right|-\left|\rho_{k+1}(g)-\rho_{k}(g)\right| \\
& \quad \geqq\left|\rho_{k}(f)-\rho_{k}(g)\right|-a_{k}^{-1}(f)-a_{k}^{-1}(g), \quad \text { by }(6) \\
& \quad \geqq\left|\rho_{k}(f)-\rho_{k}(g)\right|-2 \alpha^{-k+n-1} a_{n-1}^{-1}(f) .
\end{aligned}
$$

Hence, we have

$$
\begin{aligned}
|\theta(f)-\theta(g)| & =\lim _{m \rightarrow \infty}\left|\rho_{m}(f)-\rho_{m}(g)\right| \\
= & \left|\rho_{n}(f)-\rho_{n}(g)\right|+\sum_{k=n}^{\infty}\left\{-\left|\rho_{k}(f)-\rho_{k}(g)\right|\right. \\
& \left.\quad+\left|\rho_{k+1}(f)-\rho_{k+1}(g)\right|\right\} \\
\geqq & \left|\rho_{n}(f)-\rho_{n}(g)\right|-\sum_{k=n}^{\infty} 2 \alpha^{-k+n-1} a_{n-1}^{-1}(f), \text { by (11) } \\
= & a_{n-1}^{-1}(f)\left(1-2(\alpha-1)^{-1}\right)>0, \quad \text { since } \alpha>3 .
\end{aligned}
$$

Let $S=\{\theta(f): f \in J\}$. If $\theta=\theta(f) \in S$, and $\left\{a_{n}\right\}=\left\{a_{n}(f)\right\}$ is the sequence defined above, we claim that $\left\{a_{n} \theta^{-n}\right\}$ converges to a number $\lambda>0$, and that

$$
\left|a_{n}-\lambda \theta^{n}\right| \leqq(\theta-1)^{-1}(\alpha-1)^{-1} \quad \text { for } n=0,1, \cdots .
$$

To prove this, first let $m \rightarrow \infty$ in (9) to obtain

$$
\left|\theta-\rho_{n}\right|<a_{n-1}^{-1}(\alpha-1)^{-1} \text {. }
$$


Multiplying (14) by $a_{n-1} \theta^{-n}$, we obtain

$$
\left|a_{n-1} \theta^{-(n-1)}-a_{n} \theta^{-n}\right|<\theta^{-n}(\alpha-1)^{-1}, \quad n \geqq 1 .
$$

Thus, $\theta>1$ together with (15) implies $\left\{a_{n} \theta^{-n}\right\}$ is a Cauchy sequence with limit $\lambda$, say, and

$$
\begin{aligned}
\left|a_{n} \theta^{-n}-\lambda\right| & \leqq \sum_{m=n}^{\infty}\left|a_{m} \theta^{-m}-a_{m+1} \theta^{-(m+1)}\right| \\
& <(\alpha-1) \sum_{m=n}^{\infty} \theta^{-m-1}=\theta^{-n}(\theta-1)^{-1}(\alpha-1)^{-1} .
\end{aligned}
$$

This establishes the estimate (13).

Since $\theta \geqq \alpha>3,(\theta-1)^{-1}(\alpha-1)^{-1}<1 / 4$ so (13) implies that

$$
\left\|\lambda \theta^{n}\right\| \leqq(\theta-1)^{-1}(\alpha-1)^{-1}, \quad n=0,1, \cdots .
$$

Note also that $\left|\lambda-a_{0}\right| \leqq 1 / 4$ so $a_{0}$ is the nearest integer to $\lambda$.

REMARKS. In order to obtain $\theta$ so that the estimate $\left\|\lambda \theta^{n}\right\|$ $\leqq(\theta-1)^{-1}(\alpha-1)^{-1}$ is of the same order of magnitude as the right member of (1), we first choose $\alpha$ large and $\beta=\alpha+2$, say, so the restriction on $a_{0}$ imposed by the theorem is $a_{0}>(\alpha+1) / 2(\alpha-1)$ or $a_{0} \geqq 1$, since $\alpha>3$. Choosing $a_{0}=2$, we have $|\lambda-2| \leqq(\theta-1)^{-1}(\alpha-1)^{-1}$ which means $\lambda>1$, and $\log \lambda<\log 2+\epsilon(\alpha)$, where $\epsilon(\alpha) \rightarrow 0$ as $\alpha \rightarrow \infty$. Thus we can obtain $\theta>1, \lambda \geqq 1$, with $\theta$ transcendental, and

$$
\left\|\lambda \theta^{n}\right\| \leqq 10(2 e \theta(\theta+1)(1+\log \lambda))^{-1} \quad n=0,1, \cdots .
$$

Of course 10 may be replaced by any number greater than $2 e(1+\log 2)$.

\section{REFERENCES}

1. J. W. S. Cassels, An introduction to diophantine approximation, Cambridge Univ. Press, New York, 1957.

2. J. F. Koksma, Ein mengentheoretischer Satz über die Gleichverteilung modulo Eins, Compositio Math. 2 (1935), 250-258.

3. C. Pisot, La répartition modulo 1 et les nombres algébriques, Ann. Scuola Norm. Sup. Pisa, 7 (1938), 205-248.

4. - Répartition (mod 1) des puissances successives des nombres réels, Comment. Math. Helv. 19 (1946), 153-160.

5. R. Salem, $A$ remarkable class of algebraic integers. Proof of a conjecture of Vijayaraghavan, Duke Math. J. 11 (1944), 103-108.

6. - Algebraic numbers and Fourier analysis, Heath, Boston, Mass., 1963.

7. H. Weyl, Über die Gleichverteilung von Zahlen modulo Eins, Math. Ann. 77 (1916), 313-352.

California Institute of Technology 